Post-print version of:

Publisher: SAGE

Journal paper: Journal of Strain Analysis for Engineering Design 2018, 53(4) 210-224

Title: Integral method coefficients for the ring-core technique to evaluate non-uniform residual stresses

Authors: M. Barsanti, M. Beghini, C. Santus, A. Benincasa, L. Bertelli

Creative Commons Attribution Non-Commercial No Derivatives License

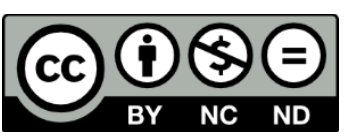

DOI Link: https://doi.org/10.1177/0309324718760438 


\title{
Integral method coefficients for the ring-core technique to evaluate non-uniform residual stresses
}

Journal Title

$\mathrm{XX}(\mathrm{X}): 1-20$

CThe Author(s) 2018

Reprints and permission:

sagepub.co.uk/journalsPermissions.nav

DOI: 10.1177/ToBeAssigned

www.sagepub.com/

(S)AGE

\author{
Michele Barsanti ${ }^{1}$, Marco Beghini ${ }^{1}$, Ciro Santus ${ }^{1}$, Alessio Benincasa ${ }^{2}$, Lorenzo \\ Bertelli $^{2}$
}

\begin{abstract}
The ring-core technique allows for the determination of non-uniform residual stresses from the surface up to relatively higher depths as compared to the hole-drilling technique. The integral method, which is usually applied to the hole-drilling, can also be used for elaborating the results of the ring-core test since these two experimental techniques share the axisymmetric geometry and the $0-45-90$ degrees layout of the strain gage rosette. The aim of this paper is at providing accurate coefficients which can be used for evaluating the residual stress distribution by the ring-core integral method. The coefficients have been obtained by elaborating the results of a very refined plane harmonic axisymmetric finite element model and verified with an independent 3D model. The coefficients for small depth steps were initially provided, then the values for multiple integer step depths were also derived by manipulating the high resolution coefficient matrices, thus showing how the present results can be practically used for obtaining the residual stresses according to different depth sequences, even non-uniform. This analysis also allowed the evaluation of the eccentricity effect which turned out to be negligible due to the symmetry of the problem. An applicative example was reported in which the input of the experimentally measured relaxed strains were elaborated with different depth resolutions, and the obtained residual stress distributions compared.
\end{abstract}

\section{Keywords}

integral method coefficients, depth resolution, eccentricity sensitivity, FE modelling, ring-core.

\footnotetext{
${ }^{1}$ Dept. of Civil and Industrial Engineering (DICI), University of Pisa, Italy

${ }^{2}$ SINT Technology Srl, Florence, Italy

Corresponding author:

Ciro Santus, University of Pisa, DICI dept. Largo Lucio Lazzarino 1, 56122 Pisa, Italy

Email: ciro.santus@ing.unipi.it
} 


\section{Introduction}

The hole-drilling and the ring-core are semi-destructive mechanical techniques used for determining residual stresses at the surface and in the near surface regions, in components with a locally flat surface. Both techniques are based on axisymmetric material removal. The hole-drilling is a well established procedure defined by internationally accepted standards ${ }^{1 ; 2}$. The ring-core was introduced more than 20 years ago $^{3-6}$, however it was deeply investigated and developed only recently, especially in terms of sensitivity and uncertainty analysis $^{7-10}$, and even applied at the microscale ${ }^{11 ; 12}$. In the hole-drilling the material is removed at the centre of a rosette thus the relaxed strains are measured at the periphery of the hole, whereas in the ringcore the strains are measured in the central internal region, as shown in Figure 1. These two techniques can be considered complementary. The hole-drilling is more popular being dedicated to measure the residual stresses near the surface ${ }^{13-18}$ (typically in the layer up to $1 \mathrm{~mm}$, or slightly larger) while the ring-core technique, having a large groove diameter ${ }^{19}$, is usually suitable for larger size components where the residual stresses at depths in the order of a few millimetres are of interest, such as rotor forging ${ }^{20}$ or thick welded plates ${ }^{21}$.

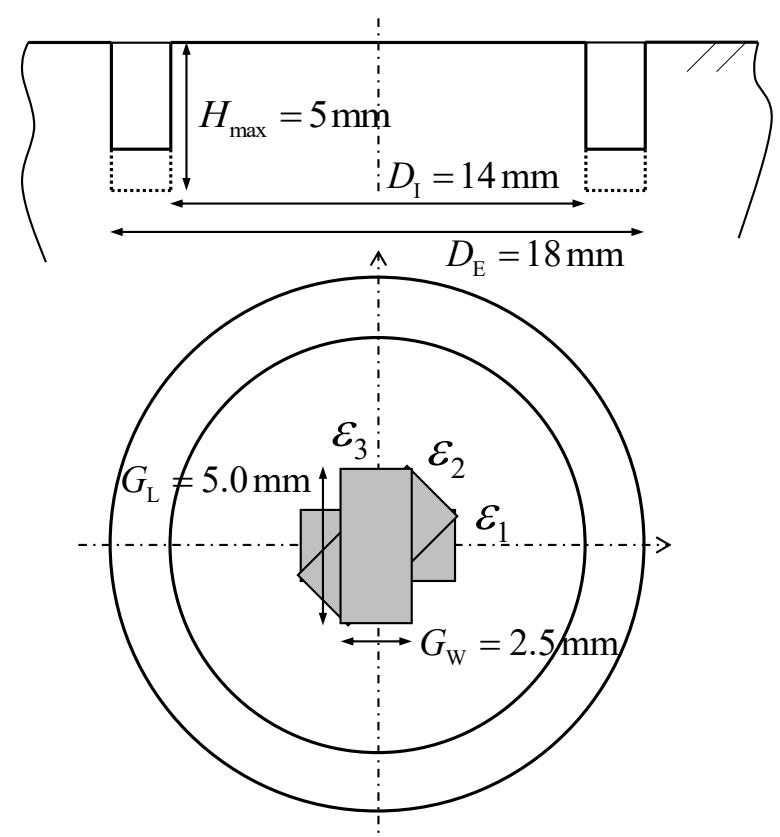

Figure 1. Ring-core technique, typical dimensions of the circular groove and strain gage grids.

Regarding the hole-drilling, the calculation of the residual stresses, after having produced a hole in incremental depths and the relieved strains recorded, can be performed with the standard ASTM E837 - 13a ${ }^{1}$ that applies the integral method. The same calculation procedure can be applied to elaborate the relaxed strains produced by the ring-core ${ }^{8 ; 10 ; 22}$, since the axial-symmetry of the problem and the grid layout according to the $0-45-90$ degrees scheme are the same. However, other numerical techniques for the residual stress determination have been proposed, such as the incremental strain method ${ }^{21 ; 23 ; 24}$ or the influence function analytical technique as proposed by Beghini et al. ${ }^{13 ; 14}$ for the hole-drilling. 
This paper presents an accurate Finite Element (FE) analysis, based on the plane harmonic axisymmetric elements, by which the coefficients for applying the integral method to the ring-core were obtained. It is worth noting that the use of a plane model with harmonic elements for solving this problem does not imply any approximation as the element type captures exactly the angular dependence of the solution. This FE approach is highly recommended for a parametric analysis of this type as dramatically reduces the number of nodes and elements if compared to a 3D model with equivalent level of accuracy. Recently, Salvati et al. ${ }^{12}$ used an $^{\text {a }}$ axisymmetric model to interpret the equibiaxial component in a micro ring-core measurement. However, as shown by Barsanti et al. ${ }^{25}$ for the hole-drilling method, the axisymmetric elements with the harmonic feature allows to model the shear components too and consequently any in-plane stress condition. In other words, the (simple) axisymmetric element type allows the determination of the equibiaxial matrix $\overline{\mathbf{a}}$, while with the harmonic axisymmetric elements both matrices $\overline{\mathbf{a}}$ and $\overline{\mathbf{b}}$ can be obtained.

The ring-core geometrical parameters are shown in Figure 1 where the grid dimensions reproduce the HBM RY51 rosette. The aim of this paper is to obtain and provide the coefficients for a sequence of small depth steps $(\Delta H=0.1 \mathrm{~mm})$ in order to give the possibility to derive, with simple calculations, the coefficients for larger steps too. The consistency of the results was validated with a 3D FE model completely independent from the reference axisymmetric model used for the calculation of the proposed coefficients. Finally, an applicative example illustrates the practical use of the provided coefficients.

\section{Outline of the integral method}

For a plane problem under a uniform residual stress field and according to the hypothesis that the grid centre belongs to the cylindrical groove axis, the relationship relating the measured relaxed strain $\varepsilon_{\mathrm{r}}$ to the principal (residual) stresses, as introduced by Schajer ${ }^{26 ; 27}$, is:

$$
\varepsilon_{\mathrm{r}}(\vartheta)=A\left(\sigma_{\max }+\sigma_{\min }\right)+B\left(\sigma_{\max }-\sigma_{\min }\right) \cos (2 \vartheta)
$$

where $\sigma_{\max }$ and $\sigma_{\min }$ are the maximum and minimum residual normal stresses, respectively, $\vartheta$ is the angle between the principal direction of $\sigma_{\max }$ and the axis of the grid, and $A, B$ are two constants depending on the geometry and on the elastic material properties $E$ and $v$. From equation (1), the following matrix relationship is obtained:

$$
\left[\begin{array}{ccc}
(A+B) & (A-B) & 0 \\
A & A & 2 B \\
(A-B) & (A+B) & 0
\end{array}\right]\left[\begin{array}{l}
\sigma_{1} \\
\sigma_{3} \\
\tau_{13}
\end{array}\right]=\left[\begin{array}{l}
\varepsilon_{1} \\
\varepsilon_{2} \\
\varepsilon_{3}
\end{array}\right]
$$

in which the subscripts $1,2,3$ are related to the three grid directions according to the 0-45-90 degrees scheme and then 1 and 3 are two orthogonal directions which can be taken as the local reference frame, Figure 1. As the directions 1 and 3 are in general not coincident with the residual stress principal directions, the shear stress component $\tau_{13}$ can be nonzero. The form of equation 2 suggests that three scalar relationships can be written in a decoupled form, after introducing three strains and three stresses new variables which are linear combinations of the reference frame strain and stress components. The definitions of these variables 
can be retrieved in the standard ASTM E837, and the following quantities are needed to be introduced for the measured strains:

$$
p=\frac{\varepsilon_{3}+\varepsilon_{1}}{2} \quad q=\frac{\varepsilon_{3}-\varepsilon_{1}}{2} \quad t=\frac{2 \varepsilon_{2}-\left(\varepsilon_{3}+\varepsilon_{1}\right)}{2}=\varepsilon_{2}-p
$$

in which $p$ is the equibiaxial and $q$ and $t$ are the shear components, and the sign of $t$ is discussed below. The residual stresses can be similarly combined, thus defining an equibiaxial $P$ and two shear components $Q, T$ :

$$
P=\frac{\sigma_{3}+\sigma_{1}}{2} \quad Q=\frac{\sigma_{3}-\sigma_{1}}{2} \quad T=\tau_{13}
$$

After defining the combined strains $p, q, t$ and combined stresses $P, Q, T$, equation 2 can be rewritten as three decoupled relations:

$$
\left[\begin{array}{ccc}
2 A & 0 & 0 \\
0 & 2 B & 0 \\
0 & 0 & 2 B
\end{array}\right]\left[\begin{array}{l}
P \\
Q \\
T
\end{array}\right]=\left[\begin{array}{l}
p \\
q \\
t
\end{array}\right]
$$

By introducing the material Young's modulus and the Poisson's ratio, the relationship available in the ASTM standard ${ }^{1}$ can be obtained:

$$
-\frac{1}{E}\left[\begin{array}{ccc}
a(1+v) & 0 & 0 \\
0 & b & 0 \\
0 & 0 & b
\end{array}\right]\left[\begin{array}{l}
P \\
Q \\
T
\end{array}\right]=\left[\begin{array}{l}
p \\
q \\
t
\end{array}\right]
$$

where the dimensionless positive coefficients $a$ and $b$ only depend on the ratios between the groove diameters and the grid dimensions. In principle, $a$ and $b$ are unaffected by the Poisson's ratio $v$ only for a plane stress model, i.e. for a through-thickness hole geometry, while this is not true in a general three-dimensional problem. The Poisson's ratio dependence in equation (6) is therefore only approximate. In fact, the coefficients are functions of the Poisson's ratio, $a(v)$ and $b(v)$, however in the range $0.25<v<0.35$ the differences are in the order of a few percent, thus it is usually assumed: $a=a(0.3)$ and $b=b(0.3)$.

When the residual stresses vary along the depth direction, the same approach can be followed but a vectorial form is required. If the circular groove is performed in $n$ steps, usually each with the same depth $\Delta H$, the scalars $p, q, t$ and $P, Q, T$ are replaced by $n$-dimensional vectors:

$$
\begin{aligned}
\mathbf{p} & =\left(p^{(1)}, p^{(2)}, \ldots, p^{(n)}\right)^{\mathrm{T}} \\
\mathbf{q} & =\left(q^{(1)}, q^{(2)}, \ldots, q^{(n)}\right)^{\mathrm{T}} \\
\mathbf{t} & =\left(t^{(1)}, t^{(2)}, \ldots, t^{(n)}\right)^{\mathrm{T}}
\end{aligned}
$$

and

$$
\begin{aligned}
& \mathbf{P}=\left(P^{(1)}, P^{(2)}, \ldots, P^{(n)}\right)^{\mathrm{T}} \\
& \mathbf{Q}=\left(Q^{(1)}, Q^{(2)}, \ldots, Q^{(n)}\right)^{\mathrm{T}} \\
& \mathbf{T}=\left(T^{(1)}, T^{(2)}, \ldots, T^{(n)}\right)^{\mathrm{T}}
\end{aligned}
$$

which represent the combined strains and stresses, respectively, at each $i$-th depth step: $i=1, \ldots, n$. The general $p^{(i)}$ term represents the combined relaxed strain (equation 3) measured when the groove depth is $i \times \Delta H$, while $P^{(i)}$ represents the combined stress that is assumed to be uniform from the depth $(i-1) \times \Delta H$ to $i \times \Delta H$. Similar 
definitions are valid for $q^{(i)}, t^{(i)}$ and shear stresses $Q^{(i)}, T^{(i)}$. Consequently, the scalars $a$ and $b$ in equation 6 have to be replaced by $n \times n$ lower triangular matrices $\overline{\mathbf{a}}$ and $\overline{\mathbf{b}}$. By combining the definitions and the relations introduced above, the matrix form of the integral method is:

$$
-\frac{1+v}{E} \overline{\mathbf{a}} \mathbf{P}=\mathbf{p} \quad-\frac{1}{E} \overline{\mathbf{b}} \mathbf{Q}=\mathbf{q} \quad-\frac{1}{E} \overline{\mathbf{b}} \mathbf{T}=\mathbf{t}
$$

When the matrices $\overline{\mathbf{a}}, \overline{\mathbf{b}}$ are available, the residual stress distribution of any experimental case can be deduced from the measured strains by solving the linear systems 9 . The elements $a_{i j}, b_{i j}$ of the just defined calibration matrices for the ring-core are calculated and provided in the next section.

A discussion about the sign of the combined strain $t$ has been already provided by Barsanti et al. ${ }^{25}$ for the holedrilling, and it is reconsidered here for the ring-core as it can be a source of formal errors in the elaboration. After introducing the orientations of the directions 1 and 3 , if the second grid is along the bisector of a quadrant where the coordinates have same sign (1st or 3rd quadrant), Figure 2, and the residual shear $\tau_{13}$ is positive, a negative (contraction) strain is measured by the second grid after introducing the groove. Assuming no equibiaxial strain component, the combined strain $t$ is equal to $\varepsilon_{2}$, equation (3), thus it is negative too. According to equation (9), the combined stress $T$, that is equal to the shear stress $\tau_{13}$, and the combined strain $t$ have opposite sign, thus confirming the accurate definition of the last of equation (3). On the contrary, if the second grid is aligned with the bisector of a quadrant with opposite sign coordinates (2nd or 4th quadrant) the second grid strain and the shear stress have the same sign, and then the opposite definition of the last of equation (3) is required, in agreement with the ASTM standard. However, the scheme with the second grid along the bisector of the 1st or the 3rd quadrant is preferred in the present work, thus the sign of the last of equations 3 is confirmed.

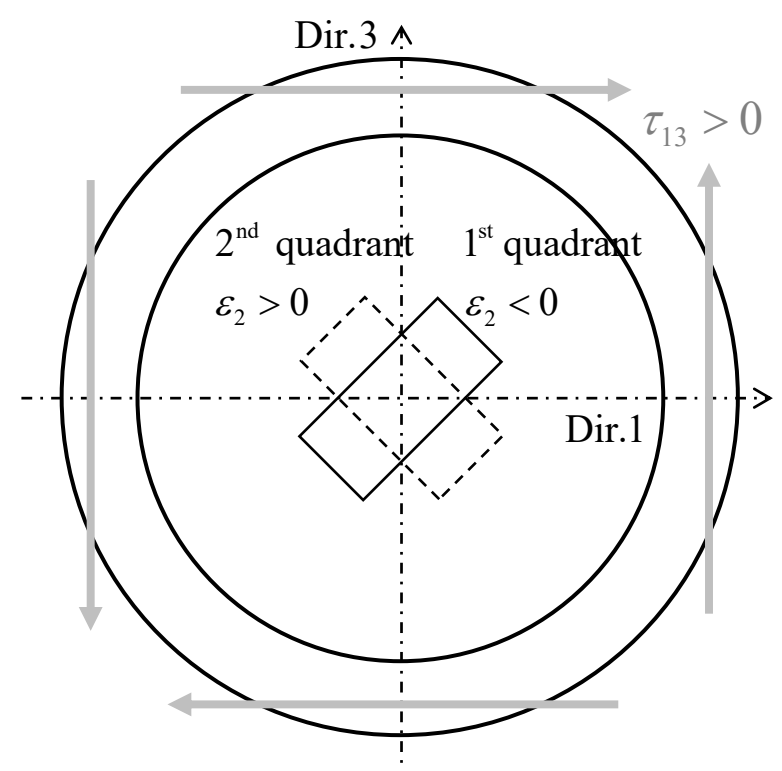

Figure 2. Scheme for deducing the sign of the second grid strain with respect to its angular position. 


\section{Axisymmetric harmonic FE model}

A refined plane FE model was generated with 5 nested regions having different nodal density. The element size was reduced by a factor of two when passing from one region to the internal one, in order to have the innermost zone, where the material removal is simulated, with $0.1 \mathrm{~mm}$ square-shaped elements, and this element edge was 70 times smaller than the internal radius of the groove, Figure 3(a). The FE model height and width were chosen much larger than the groove internal radius, Figure 3(a), to reproduce the condition of a virtually semiinfinite body. The far field boundary conditions influenced the simulated displacements within the groove, and the calculated coefficients, with an estimated effect in the order of $10^{-3}$. Approximately 126000 axisymmetric harmonic elements were used, and the element type was ANSYS® Plane25.

In agreement with equation (9), the general state of residual stress was represented by superimposing an equibiaxial stress and two pure shears. The equibiaxial and the pure shear stress components were applied as two independent load steps, Figure 3(b). Only a single shear stress component was actually required to be modelled, as the matrix to be determined is just $\overline{\mathbf{b}}$ for both shears. The equibiaxial load was modelled with a zero order harmonic analysis, i.e. a radial pressure constant in the angular direction, applied on the cylindrical surfaces of the groove. Whereas, the shear load was obtained by superimposing a normal and a tangential traction distributions, both as second order harmonics with $2 \vartheta$ variation, relatively shifted by an angle of $45^{\circ}$, Figure 3(b).
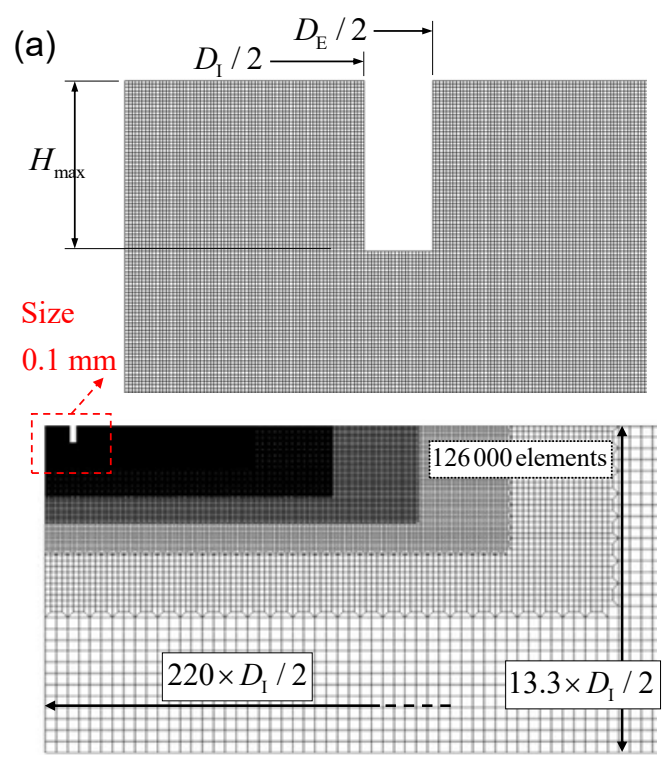

(b)
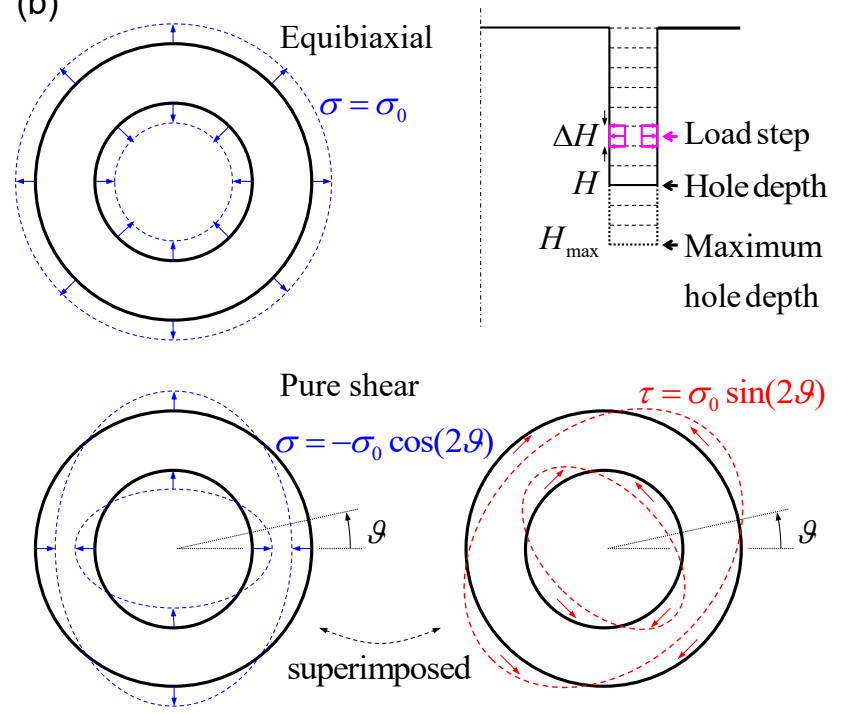

Figure 3. (a) Harmonic plane axisymmetric FE model. (b) Scheme of the basis loading conditions applied to the FE model.

Similarly to the simulations for the hole-drilling technique ${ }^{25}$, the FE model is residual stress free, the material at the groove is preliminarily removed and the proper tractions are applied to both the inner and outer cylindrical surfaces. Actually, the physical problem is the opposite. Residual stresses are pre-existing, so the material removal relaxes to zero the tractions at the surfaces of the groove. In order to take into account this 
alternative way of modelling, a minus sign has to be introduced in the relation between stresses and strains. In fact, by applying the traction at the free surfaces, the opposite strain is obtained with respect to the theoretical removal of the material.

The same resolution of the model geometry was applied to the integral method load step, thus $\Delta H=0.1 \mathrm{~mm}$. For example, when the groove had depth $H=4.2 \mathrm{~mm}, 42$ load positions were analysed, the first with the load from 0.0 to $0.1 \mathrm{~mm}$, then from 0.1 to $0.2 \mathrm{~mm}$, and so on. Since the maximum considered groove depth was $H_{\max }=5.0 \mathrm{~mm}$, the total number of single simulations (equibiaxial and pure shear) was: $2 \times(50 \times(50+1) / 2)=2550$.

\section{Coefficients for the integral method}

\section{Coefficient derivation from the displacement fields}

The coefficients of the matrices $\overline{\mathbf{a}}, \overline{\mathbf{b}}$ can be derived by imposing a single unitary traction at each depth position, as described in the previous section, and calculating with the FE results the combined strains virtually measured by the rosette. For instance, to calculate the $a_{i j}$ element, the stress components $P^{(j)}=1$, $P^{(k, k \neq j)}=0$ were imposed, and the strain component $p_{i}$ calculated. The simulated strain measured by the grids was evaluated by computing the average displacement (in the grid direction) at the extreme edge segments, and dividing the averaged displacement differences by the grid length, without retrieving any displacement information at the intermediate positions of the grids. With reference to Figure 4, the whole procedure is summarized in detail hereafter:

1. Define the vertices $A, B, C, D$ of each grid and a large enough number of integration points (about 100) on the sides $A B$ and $C D$, both in cartesian and polar coordinates;

2. Introduce the components of the unit vectors transverse $\hat{\mathbf{t}}$ and normal $\hat{\mathbf{n}}$ (external to the grid) to the segments $A B$ and $C D$, at each integration point;

3. Calculate the displacements along the radial and the tangential directions by local linear interpolation of the FE element results, at each integration point on sides $A B$ and $C D$;

4. Evaluate the displacement components along the transverse and normal directions at the integration points;

5. Being the grid mainly sensitive to the extensional strain in the normal direction (transverse sensitivity, though not zero, is quite small and usually neglected) only the displacements along the $\hat{\mathbf{n}}$ direction at the integration points were considered. However, $\hat{\mathbf{t}}$ direction displacements could also be taken into account on the lateral sides $B C$ and $A D$ if the transversal strain sensitivity were significant.

6. Average the normal displacements on the active sides $A B$ and $C D$.

7. Compute the difference between $A B$ and $C D$ averaged displacements and divide it by the grid length to obtain the simulated measured strains $\varepsilon_{1}, \varepsilon_{3}$.

8. Evaluate $\varepsilon_{1}, \varepsilon_{3}$ to calculate the combined strains $p$ and $q$, while $\varepsilon_{2}$ is unnecessary since the shear applied according to the load scheme of Figure 3 (b) is not along the 45 degrees direction.

9. Deduce the matrix coefficients by multiplying $p_{i}$ by $-E /(1+v)$ for $a_{i j}$, and $q_{i}$ by $-E$ for $b_{i j}$. 

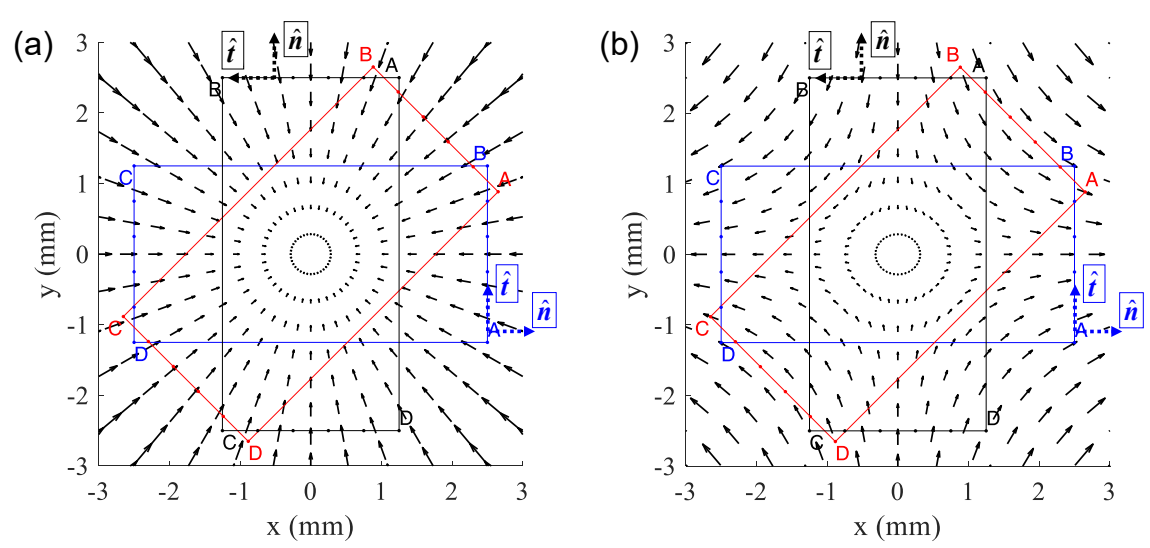

Figure 4. Displacement fields on the strain gage areas: (a) equibiaxial, and (b) pure shear load cases.

This calculation was repeated for all the 2550 simulated groove depths, load depths and load type combinations to obtain the coefficients $a_{i j}, b_{i j}$, with $i \geq j$ (coefficients with $i<j$ are zero). The $\overline{\mathbf{a}}, \overline{\mathbf{b}}$ matrices obtained for the Poisson's ratio $v=0.3$ are reported in Tables 1 and 2, split in blocks to fit the paper page, and also electronically available on the online Appendix (http://sdj.sagepub.com).

\section{Integer multiple step coefficient determination}

Lower resolution coefficients can be derived for any $m$ multiple depth step of $\Delta H=0.1 \mathrm{~mm}$, for instance $m=2,5,10$ in order to be applied to measurements with higher depth steps. By implementing the superposition principle, the lower resolution coefficients can be obtained as simple summations of the original coefficients: one every $m$ rows must be taken into account, corresponding to $m \times 0.1 \mathrm{~mm}$ groove depth. For each of these rows, elements must be collected in blocks of size $m$ (starting from the leftmost one), and all the elements in the same block must be summed. At the end of this procedure, two smaller lower triangular matrices of size $(50 / m) \times(50 / m)$ are obtained.

For example, in order to obtain $a_{i j}$ and $b_{i j}$ for $1 \mathrm{~mm}$ depth step ( $\left.m=10\right)$, only the row indices 10, 20, 30, 40, 50 must be considered. In the row with $i=10$ of the matrix $\overline{\mathbf{a}}$, a single block of 10 values is built, whose sum is 0.1145 . In the row with $i=20$ two blocks of 10 values are built, whose sums are 0.1966 and 0.1139 , and so on up to the row with $i=50$ in which 5 values are obtained by summing the elements contained in each of the 5 blocks. The same procedure is to be repeated for the matrix $\overline{\mathbf{b}}$.

Non-uniform steps, for example as initially proposed by Zuccarello ${ }^{6}$, and recently re-proposed by Menda et al. ${ }^{8 ; 9}$ and Zuccarello et al. ${ }^{10}$, can be also obtained from the large matrices introduced above. However, the depths need to be approximated with the $0.1 \mathrm{~mm}$ resolution. At the beginning, the depth $0.6 \mathrm{~mm}$ is equivalent to 6 steps of the the high resolution matrices, while $1.05 \mathrm{~mm}$ needs to be approximated as $1.1 \mathrm{~mm}$, corresponding to 11 steps. According to this scheme, as graphically shown in Figure 5, the coefficient $a_{11}\left(b_{11}\right)$ is the sum of all the six elements of the sixth row, then the coefficient $a_{21}\left(b_{21}\right)$ is the sum of the first six elements of the eleventh row, $a_{22}\left(b_{22}\right)$ is the sum of the coefficients from the seventh to the eleventh elements, and so on. 
Table 1. $a_{i j}$ coefficients $\left(\times 10^{3}\right)$ for $D_{\mathrm{I}}=14 \mathrm{~mm}, D_{\mathrm{E}}=18 \mathrm{~mm}, G_{\mathrm{L}}=5.0 \mathrm{~mm}, G_{\mathrm{W}}=2.5 \mathrm{~mm}$, maximum depth $H_{\max }=5.0 \mathrm{~mm}$ and depth resolution $\Delta H=0.1 \mathrm{~mm}$.
$a_{1}{ }_{5.3290}^{3.8156008}$

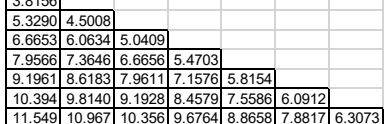

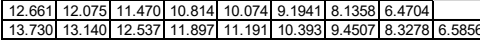

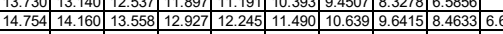

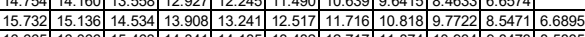

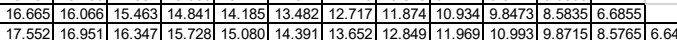

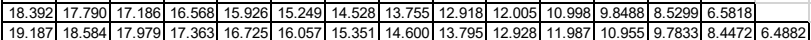

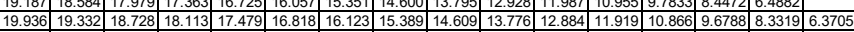

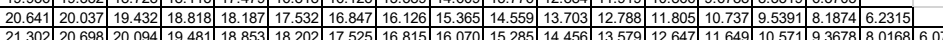

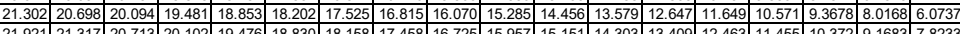

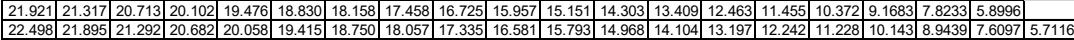

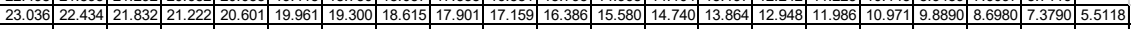

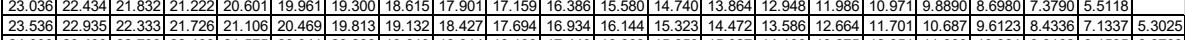

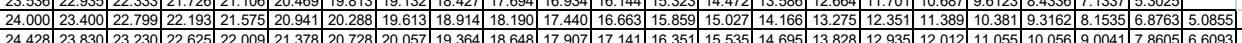

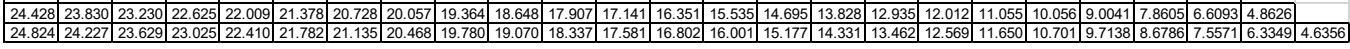

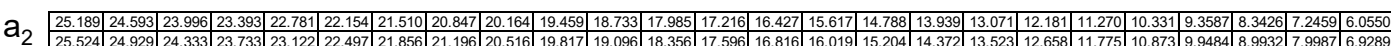

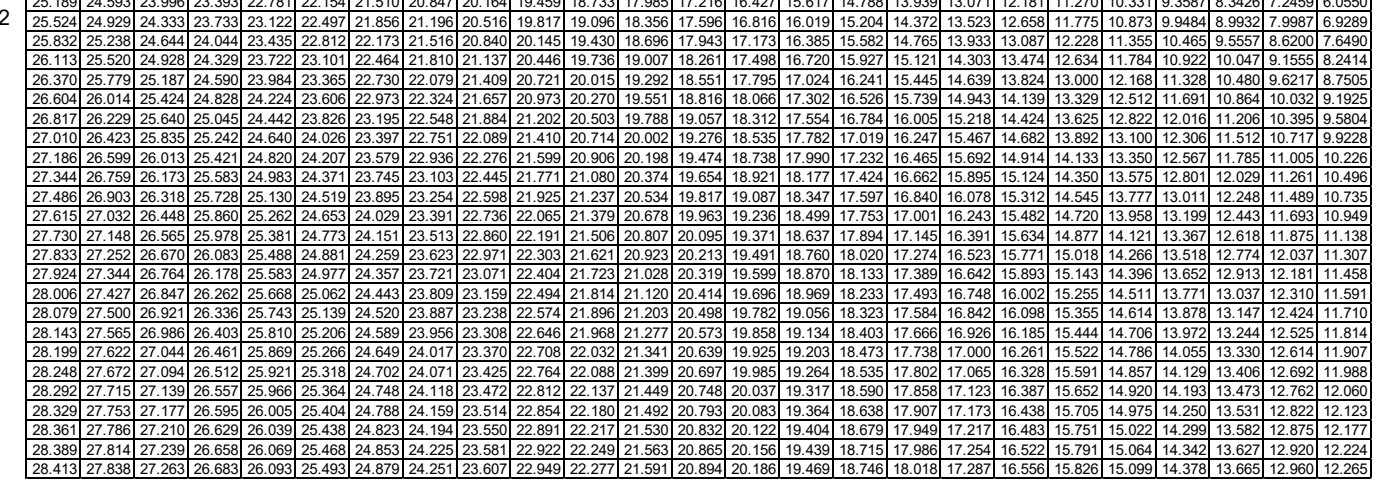

$a_{3} \frac{4.4061}{5.7715} 4.175$

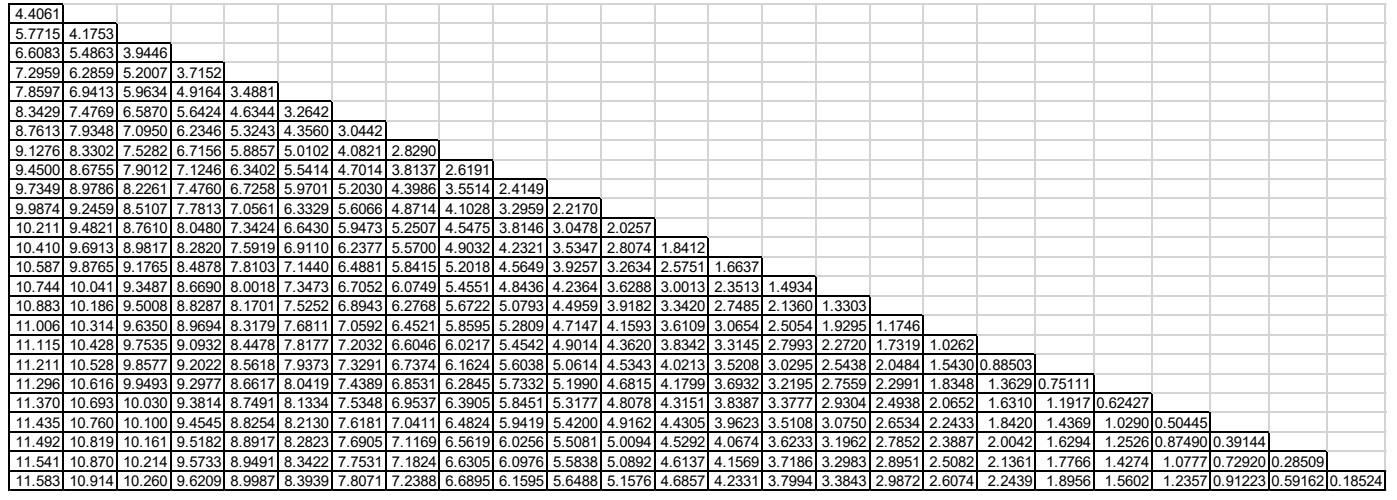

\section{Validation of the calibration coefficients}

A 3D model was implemented for validating the plane FE analysis and the proposed calculation procedure. Though coarser than the axisymmetric model, Figure 6, the load application and the relaxed strain calculation were completely different in this 3D analysis, thus it was unlikely to replicate the same error on both models. The successful comparison, even if verified for a few residual stress cases, gave high confidence about the proper application of the integral method and then the correctness of the coefficients. In principle, the same 
Table 2. $b_{i j}$ coefficients $\left(\times 10^{3}\right)$ for $D_{\mathrm{I}}=14 \mathrm{~mm}, D_{\mathrm{E}}=18 \mathrm{~mm}, G_{\mathrm{L}}=5.0 \mathrm{~mm}, G_{\mathrm{W}}=2.5 \mathrm{~mm}$, maximum depth $H_{\max }=5.0 \mathrm{~mm}$ and depth resolution $\Delta H=0.1 \mathrm{~mm}$.
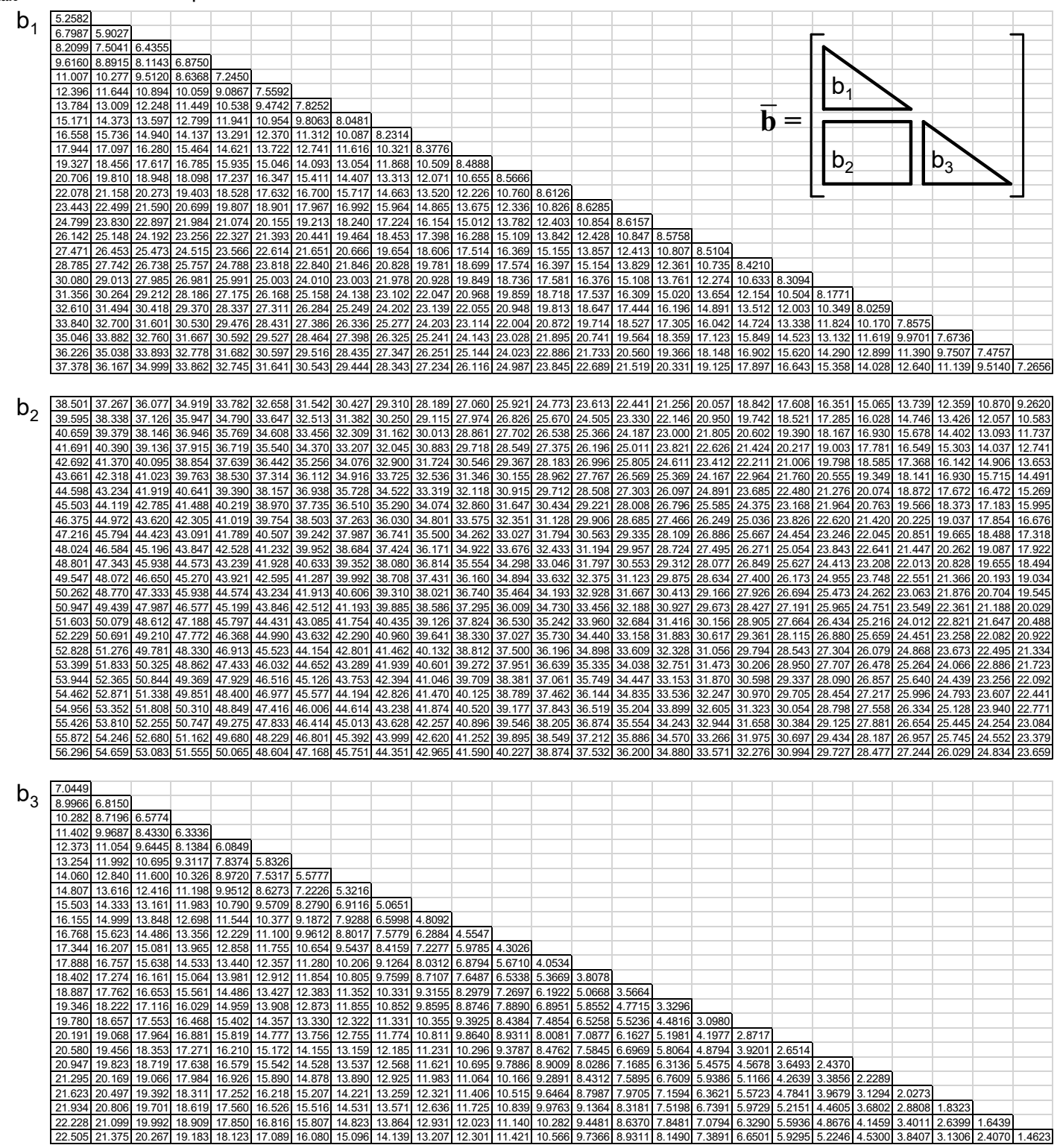

level of accuracy would have been obtained with a 3D model just by replicating the discretization in the section plane and then introducing a large number of divisions, for example 100, along the angular direction. However, such a higher number of elements would require huge computing performances, basically without any significant advantage.

In the 3D analyses, the external load was applied as far field along the two principal directions $x$ and $y$ and the material removal simulated with no traction applied to the groove surfaces. The relaxed strains were then 


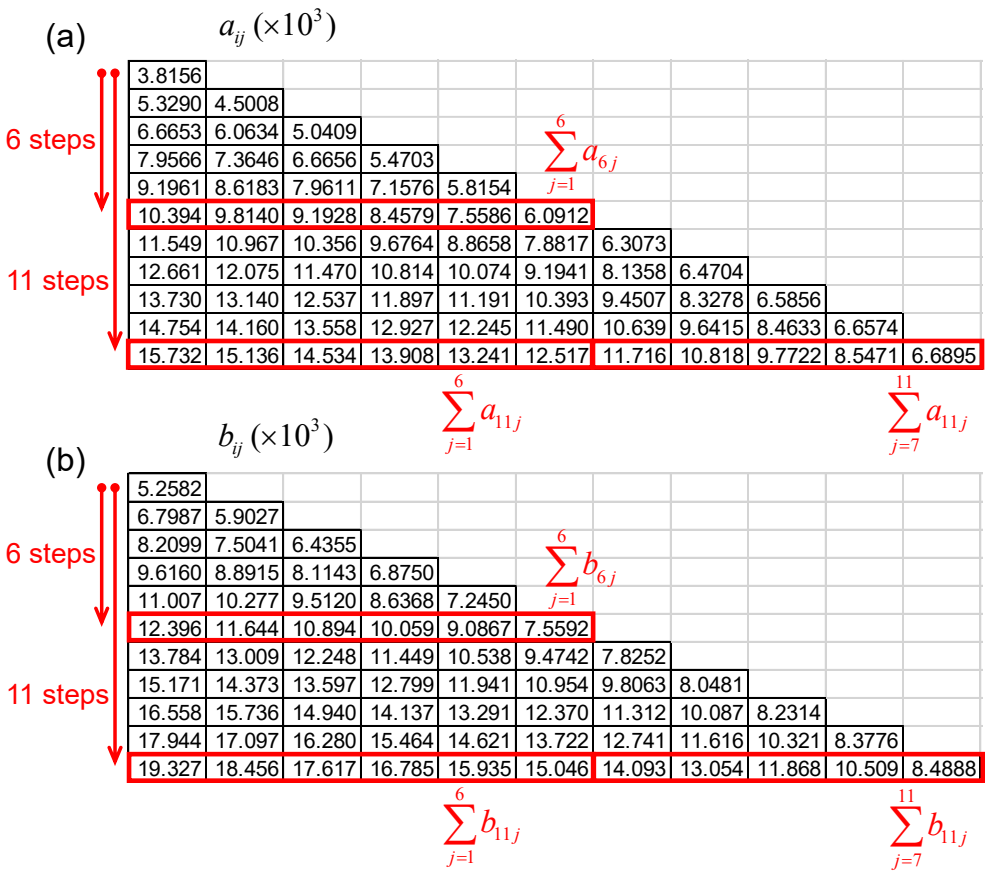

Figure 5. Calculation scheme with non-uniform depths for deriving the lower resolution coefficients of matrices $a_{i j}$ (a) and $b_{i j}$ (b).

(b)

$\sigma_{y}$

(a) $\uparrow \uparrow \uparrow \uparrow \uparrow \uparrow \uparrow \uparrow \uparrow \uparrow \uparrow$

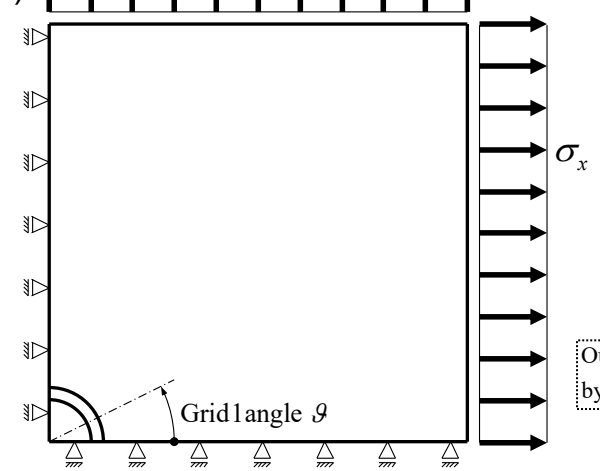

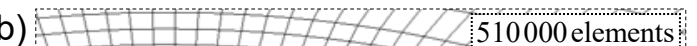

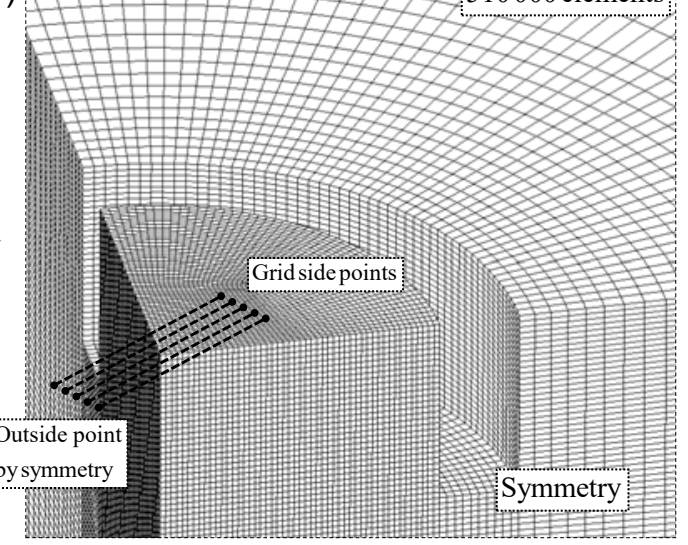

Figure 6. FE model for validation, (a) load scheme, (b) 3D mesh grid side points.

obtained as the difference between the final grooved geometry and the load uniformly applied before the material removal, which could be computed with the Hooke's law. The problem features two symmetry planes which were considered to reduce the modelled volume by a factor of 4 . The grid side points were simulated as any angle with respect to the first principal direction $x$ and those points outside the quarter volume were computed by exploiting the symmetries of the problem. The matrices for the integral method according to 0.5 
$\mathrm{mm}$ resolution of the 3D model, were obtained by applying the procedure introduced in the previous section, and reported in Table 3. Equations (9) were inverted to obtain the stresses, assuming as input the strains of the 3D FE analysis which was treated as a virtual residual stress experiment.

Table 3. Calibration coefficients with resolution $\Delta H=0.5 \mathrm{~mm}$ for the validation analysis.

\begin{tabular}{llllllllll}
\hline \multicolumn{1}{l}{$a_{i j}$ coefficients $\left(\times 10^{3}\right)$} & \multicolumn{1}{l}{$l$} & & \\
\hline 38.75 & 0 & 0 & 0 & 0 & 0 & 0 & 0 & 0 & 0 \\
67.64 & 46.89 & 0 & 0 & 0 & 0 & 0 & 0 & 0 & 0 \\
89.84 & 72.73 & 47.66 & 0 & 0 & 0 & 0 & 0 & 0 & 0 \\
106.4 & 90.14 & 70.30 & 43.64 & 0 & 0 & 0 & 0 & 0 & 0 \\
118.1 & 102.2 & 83.90 & 62.71 & 36.92 & 0 & 0 & 0 & 0 & 0 \\
125.9 & 110.3 & 92.68 & 73.15 & 52.35 & 29.17 & 0 & 0 & 0 & 0 \\
130.8 & 115.4 & 98.21 & 79.45 & 60.16 & 41.14 & 21.54 & 0 & 0 & 0 \\
133.8 & 118.5 & 101.5 & 83.20 & 64.60 & 46.80 & 30.43 & 14.71 & 0 & 0 \\
135.4 & 120.3 & 103.4 & 85.32 & 67.07 & 49.82 & 34.35 & 20.97 & 9.004 & 0 \\
136.3 & 121.2 & 104.4 & 86.43 & 68.37 & 51.38 & 36.29 & 23.52 & 13.12 & 4.485 \\
\hline \hline$b_{i j}$ coefficients $\left(\times 10^{3}\right)$ & & & & & & & \\
\hline 46.68 & 0 & 0 & 0 & 0 & 0 & 0 & 0 & 0 & 0 \\
81.41 & 56.78 & 0 & 0 & 0 & 0 & 0 & 0 & 0 & 0 \\
114.6 & 90.99 & 60.67 & 0 & 0 & 0 & 0 & 0 & 0 & 0 \\
146.2 & 120.6 & 93.39 & 59.51 & 0 & 0 & 0 & 0 & 0 & 0 \\
175.2 & 147.2 & 119.2 & 89.35 & 54.59 & 0 & 0 & 0 & 0 & 0 \\
200.7 & 170.4 & 140.9 & 111.0 & 80.65 & 47.29 & 0 & 0 & 0 & 0 \\
222.3 & 190.0 & 159.0 & 128.4 & 98.37 & 69.24 & 38.90 & 0 & 0 & 0 \\
240.1 & 206.0 & 173.7 & 142.2 & 111.9 & 83.35 & 56.81 & 30.39 & 0 & 0 \\
254.4 & 218.8 & 185.3 & 153.0 & 122.3 & 93.71 & 67.79 & 44.58 & 22.43 & 0 \\
265.7 & 228.8 & 194.4 & 161.4 & 130.2 & 101.5 & 75.61 & 52.96 & 33.34 & 15.37 \\
\hline
\end{tabular}

The calculated stress components were finally compared with the stresses imposed to the 3D model, after applying a tensor rotation to obtain the components according to the 1,3 grid rosette frame. Indeed, a misalignment angle was introduced between the principal stress directions and the rosette frame to have a nonzero shear stress $\tau_{13}$ which also allowed to verify the sign definition of the strain component $t$ (equation 3). Five load and angle combinations were considered and the average stress over the depth of $5 \mathrm{~mm}$ of each component was compared to the reference value, then the differences were evaluated. This comparison is shown in Table 4 in which differences not larger than 1\% are shown, and in Figure 7 where the three stress components of the last case of Table 4 are plotted. The back-calculated stress distributions resulted quite uniform with just small variations at the extremes where the method is more sensitive to the disturbance, and the slight different results of the two FE models produced a detectable effect. It is worth noting that these differences are mainly to be imputed to the coarseness of the 3D analysis, as the plane axisymmetric model features a finer mesh and an exact angular dependence. 
Table 4. Validation cases with different combinations of loads and rosette angles, and obtained small average stress differences.

\begin{tabular}{lllllll}
\hline$\sigma_{x}, \mathrm{MPa}$ & $\sigma_{y}, \mathrm{MPa}$ & $\sigma_{y} / \sigma_{x}$ & Grid $1 \vartheta$ & $\sigma_{1}$ diff. & $\sigma_{3}$ diff. & $\tau_{13}$ diff. \\
\hline 100 & 0 & 0.0 & $0^{\circ}$ & $0.1 \%$ & - & - \\
100 & 100 & 1.0 & $0^{\circ}$ & $-0.2 \%$ & $-0.2 \%$ & - \\
100 & -100 & -1.0 & $0^{\circ}$ & $0.3 \%$ & $0.3 \%$ & - \\
75 & -30 & -0.4 & $-45^{\circ}$ & $-0.2 \%$ & $-0.2 \%$ & $0.3 \%$ \\
75 & -30 & -0.4 & $20^{\circ}$ & $0.2 \%$ & $1.0 \%$ & $0.3 \%$ \\
\hline
\end{tabular}

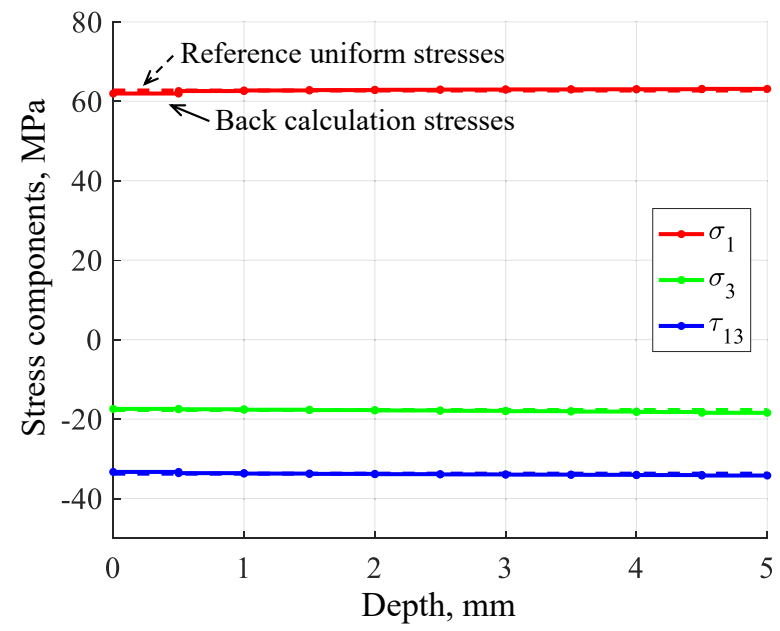

Figure 7. Comparison between the imposed and the back-calculated stresses of a validation case.

\section{Sensitivity to eccentricity}

The eccentricity between the hole and the rosette is an important issue in the hole-drilling method. The generalization of the integral method proposed by Barsanti et al. ${ }^{25}$, for taking into account of the eccentricity in the hole-drilling, was adapted to the ring-core too. According to that general approach, the $p, q, t$ and $P, Q, T$ decomposition can be no more applied as the axisymmetry of the problem is lost. A more general (still linear) relationship between all the components of stress and strain is consequently introduced:

$$
-\frac{1}{E} \overline{\mathbf{A}} \mathbf{S}=\mathbf{e}
$$

In equation (10), $\mathbf{S}=\left(\sigma_{1}^{(1)}, \sigma_{3}^{(1)}, \tau_{13}^{(1)}, \ldots, \sigma_{1}^{(n)}, \sigma_{3}^{(n)}, \tau_{13}^{(n)}\right)^{\mathrm{T}}$ is the vector collecting all the components of residual stresses in blocks of three elements (one block for each drilling depth) and $\mathbf{e}=$ $\left(\varepsilon_{1}^{(1)}, \varepsilon_{2}^{(1)}, \varepsilon_{3}^{(1)}, \ldots, \varepsilon_{1}^{(n)}, \varepsilon_{2}^{(n)}, \varepsilon_{3}^{(n)}\right)^{\mathrm{T}}$ is the vector collecting the relaxed strains, again in blocks of three 
elements. The lower triangular $3 \times 3$ block matrix $\bar{A}$ is defined in equation (11):

$$
\overline{\mathbf{A}}=\left[\begin{array}{ccc|ccc|ccc}
A_{11}^{(11)} & A_{12}^{(11)} & A_{13}^{(11)} & 0 & 0 & 0 & 0 & 0 & 0 \\
A_{21}^{(11)} & A_{22}^{(11)} & A_{23}^{(11)} & 0 & 0 & 0 & 0 & 0 & 0 \\
A_{31}^{(11)} & A_{32}^{(11)} & A_{33}^{(11)} & 0 & 0 & 0 & 0 & 0 & 0 \\
\hline A_{11}^{(21)} & A_{12}^{(21)} & A_{13}^{(21)} & A_{11}^{(22)} & A_{12}^{(22)} & A_{13}^{(22)} & 0 & 0 & 0 \\
A_{21}^{(21)} & A_{22}^{(21)} & A_{23}^{(21)} & A_{21}^{(22)} & A_{22}^{(22)} & A_{23}^{(22)} & 0 & 0 & 0 \\
A_{31}^{(21)} & A_{32}^{(21)} & A_{23}^{(21)} & A_{31}^{(22)} & A_{32}^{(22)} & A_{33}^{(22)} & 0 & 0 & 0 \\
\hline A_{11}^{(31)} & A_{12}^{(31)} & A_{13}^{(31)} & A_{11}^{(32)} & A_{12}^{(32)} & A_{13}^{(32)} & A_{11}^{(33)} & A_{12}^{(33)} & A_{13}^{(33)} \\
A_{21}^{(31)} & A_{22}^{(31)} & A_{23}^{(31)} & A_{21}^{(32)} & A_{22}^{(32)} & A_{23}^{(32)} & A_{21}^{(33)} & A_{22}^{(33)} & A_{23}^{(33)} \\
A_{31}^{(31)} & A_{32}^{(31)} & A_{33}^{(31)} & A_{31}^{(32)} & A_{32}^{(32)} & A_{33}^{(32)} & A_{31}^{(33)} & A_{32}^{(33)} & A_{33}^{(33)}
\end{array}\right]
$$

The elements $A_{h k}^{(i j)}$ have indices $h, k=1, \ldots, 3, i=1, \ldots, n$ and $j=1, \ldots, i$, where $n$ is the number of drilling steps. Each coefficient of this matrix depends on the eccentricity components $e_{1}$ and $e_{3}$ defined in the frame of the grid with axes in the directions 1 and 3 as shown in Figure 8. A power series expansion can be used to represent the dependence of these coefficients to the eccentricity, as shown in equation (12):

$$
A_{h k}^{(i j)}=A_{0, h k}^{(i j)}+\frac{\partial A_{h k}^{(i j)}}{\partial e_{1}} e_{1}+\frac{\partial A_{h k}^{(i j)}}{\partial e_{3}} e_{3}+\frac{1}{2} \frac{\partial^{2} A_{h k}^{(i j)}}{\partial e_{1}^{2}} e_{1}^{2}+\frac{1}{2} \frac{\partial^{2} A_{h k}^{(i j)}}{\partial e_{3}^{2}} e_{3}^{2}+\frac{\partial^{2} A_{h k}^{(i j)}}{\partial e_{1} \partial e_{3}} e_{1} e_{3}+\ldots
$$

If the eccentricity is small, as it is expected in a correctly applied procedure, only the constant and the linear terms of these expansions are sufficient to approximate the effect. For the hole-drilling, both the two first order derivatives are nonzero, while these terms are zero for the ring core. The physical reason of this result is the symmetry property of the geometry. As the grids are located at the centre, a displacement of the groove either parallel or orthogonal with respect to the grid direction, produces a higher sensitivity at one side and a lower sensitivity at the opposite side, as schematically shown in Figure 8, thus inducing a compensating effect. In fact, a displacement of the grid along a direction produces the same layout as the opposite direction plus a rotation of 180 degrees, which this latter is equivalent from the stress point of view. On the contrary for the hole-drilling technique, a first order net effect results unless special compensating grids are used, as investigated by Beghini et al. ${ }^{28}$ and used by Iurea et al. ${ }^{29}$, featuring an opposite grid, connected in series, for each of the three directions.

Numerical evaluation of the elements $A_{h k}^{(i j)}$ for different eccentricities performed with the proposed model, numerically confirmed that the ring-core method does not experience sensitivity to eccentricity at the first order. Two examples of this analysis are reported in Figure 9 in which it is evident that the tangent plane of the functions $A_{h k}^{(i j)}\left(e_{1}, e_{3}\right)$ at the origin is horizontal, with typical local shapes which are either an ellipsoidal surface, Figure 9(a), or a saddle surface, Figure 9(b). For this reason, it was considered not necessary to introduce the form of equation (10) which is more cumbersome, and then the procedure based on $p, q, t$ was assumed accurate enough even with a moderate eccentricity. Indeed, as evident in Figure 9 for the examined geometry, the coefficients $A_{h k}^{(i j)}$ vary less than $1 \%$ when the eccentricity vector components $\left(e_{1}, e_{3}\right)$ are in the 
range $\pm 0.2 \mathrm{~mm}$. The effects of the second order terms in equation 12 are significant only for eccentricities which are relatively large and uncommon in typical experimental applications.
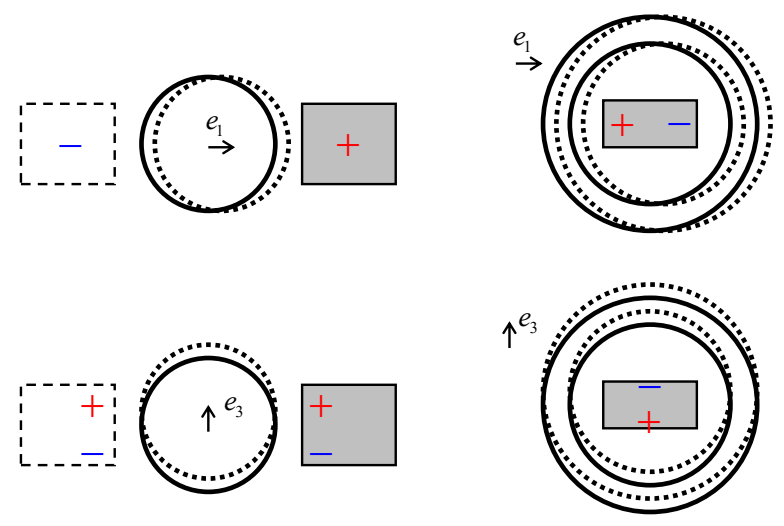

Hole drilling as reference

Ring core

Figure 8. Self compensated eccentricity sensitivity of the ring-core, comparison with the hole-drilling.

(a)

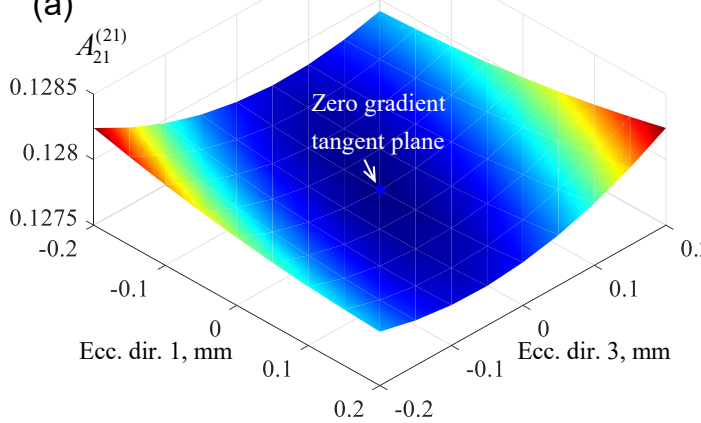

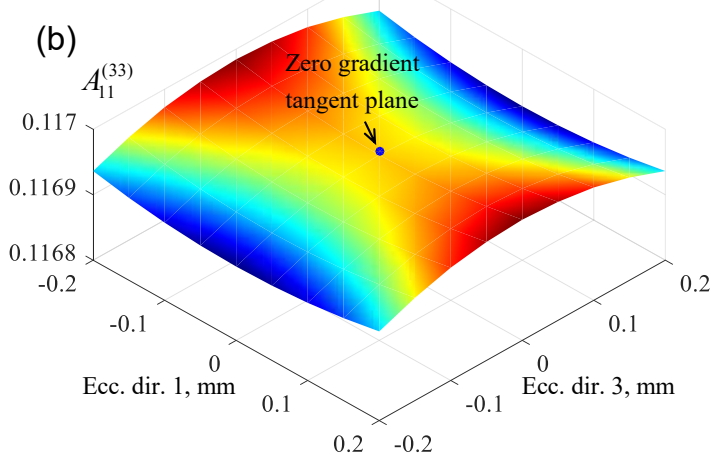

(b)

Figure 9. Eccentricity sensitivity of the general form matrix coefficients: (a) $A_{21}^{21}$, (b) $A_{11}^{33}$ examples, and zero first partial derivatives at the origin.

\section{Experimental application}

The mechanical system for automatically drilling the groove and performing the strain gage measurement, manufactured by SINT Technology ${ }^{30}$, is shown in Figure 10 along with the HBM RY51 rosette. The drilling spindle of the device is hollow thus allowing the strain gage cables to pass through. The rosette requires a special preparation to protect the grids during the drilling. The overall dimensions of the system are $310 \mathrm{~mm}$ long, $160 \mathrm{~mm}$ wide, $230 \mathrm{~mm}$ maximum height. The centring is performed with two independent mechanical micrometric guides and a control webcam, connected with Ethernet TCP/IP. By this system a positioning accuracy in the order of $\pm 0.15 \mathrm{~mm}$ can be obtained. The vertical feed motion is driven by a stepping motor, and the machining of the annular groove around the strain gage rosette is operated with a DC motor to have 
the tool rotational speed at approximately $200 \mathrm{rpm}$.

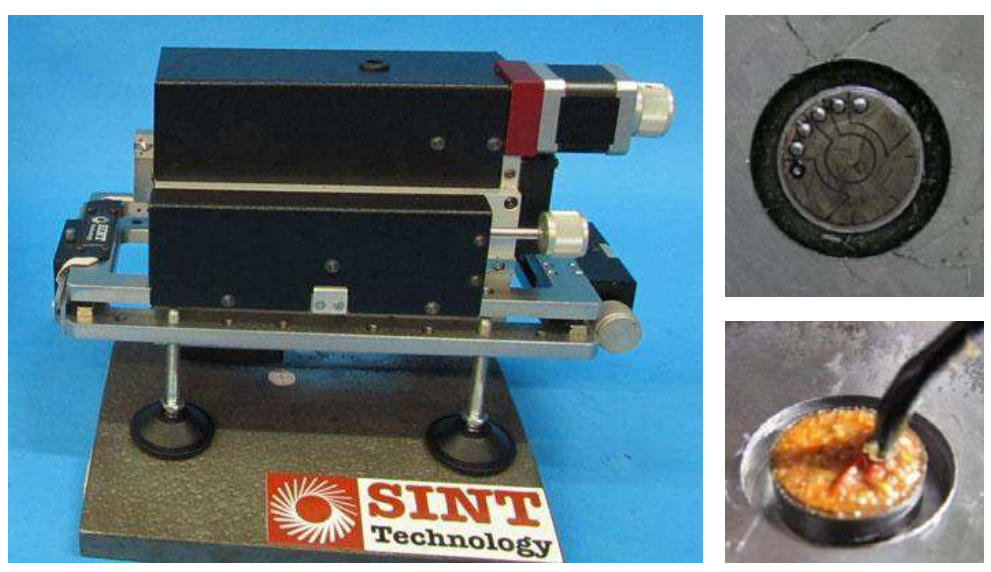

Figure 10. Equipment by SINT Technology for the ring-core automatic testing and HBM rosette.

The application introduced here is not intended to represent a validation, but an experimental example in which the residual stresses are effectively evaluated with different resolutions by means of the proposed coefficients. The ring-core rosette was applied on the lateral surface of a tubular square bar made by ASTM A500 Grade B steel carrying a longitudinal weld. The centre of the measurement region was at $40 \mathrm{~mm}$ from the weld, and the grid 1 direction perpendicular to the weld bead. The measured relaxed strains with $0.1 \mathrm{~mm}$ milling tool incremental step are plotted in Figure 11 (a). Initially, the residual stresses were calculated with two resolutions, Figure 11 (b): $1.0 \mathrm{~mm}$ (dashed lines) and $0.5 \mathrm{~mm}$ (solid lines). Higher resolution residual stress distributions, such as $0.2 \mathrm{~mm}$ or even $0.1 \mathrm{~mm}$, can also be calculated with the proposed calibration matrices. However, for these small step values, the application of a filtering technique when solving equations 9 is recommended to reduce the effect of the noise. In particular the Tikhonov regularization proposed in the standard ASTM E837, which can be applied to the integral method for the ring core in the same way as for the hole-drilling, was demonstrated by Barsanti et al. ${ }^{31}$ to mitigate the effect of the measurement noise. Finally, the non-uniform step sequence, proposed by Zuccarello ${ }^{6}$ for this arrangement, with 8 depth increments and optimized strain measurement sensitivity, was implemented by means of the coefficients derived with the calculation scheme of Figure 5. The obtained residual stress distribution is reported in Figure 11 (c), where it is evident that the trends of the components are quite similar to the solution obtained with $0.5 \mathrm{~mm}$ step. In fact, the optimized step sizes range between $0.4 \mathrm{~mm}$ and $0.7 \mathrm{~mm}$ up to $3.5 \mathrm{~mm}$ depth.

\section{Conclusions}

The paper provides the calibration coefficients to apply the integral method in the ring-core technique for measuring non-uniform in depth residual stress distributions. The harmonic plane axisymmetric finite element model used for the numerical simulation was described along with the procedure to deduce the numerically simulated measured strains. The use of a 2D FE model produced results with high resolution and accuracy. Similar results could be obtained with a more intuitive 3D model but with 2 orders of magnitude more 


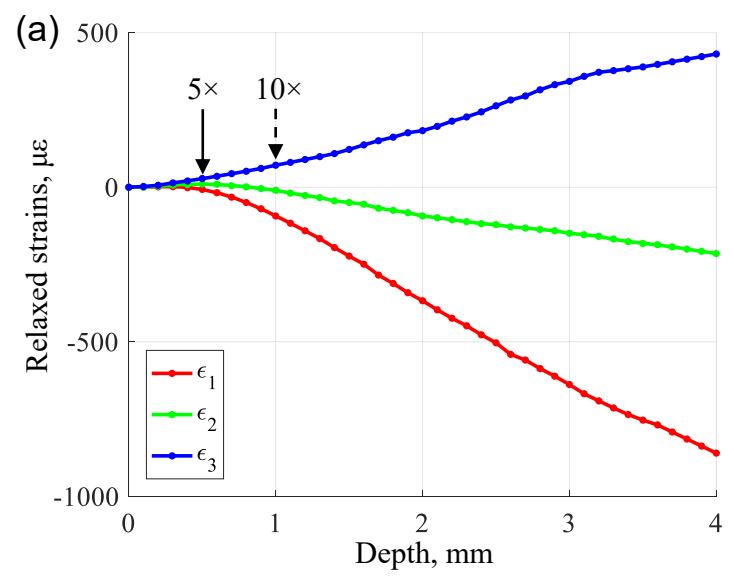

(b)

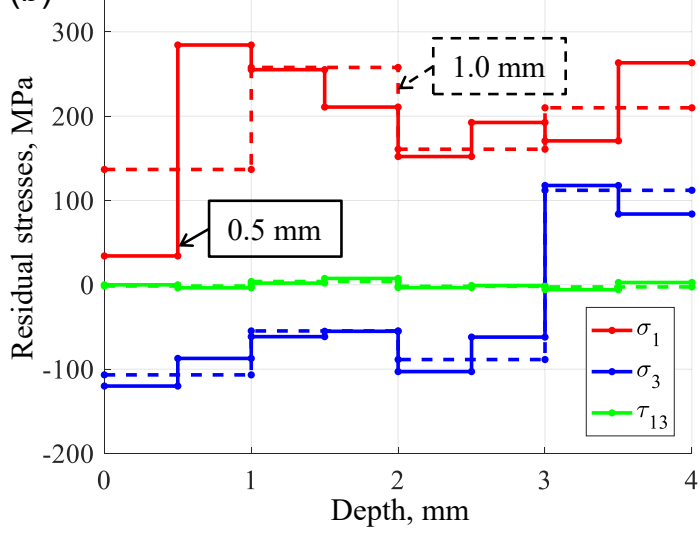

(c)

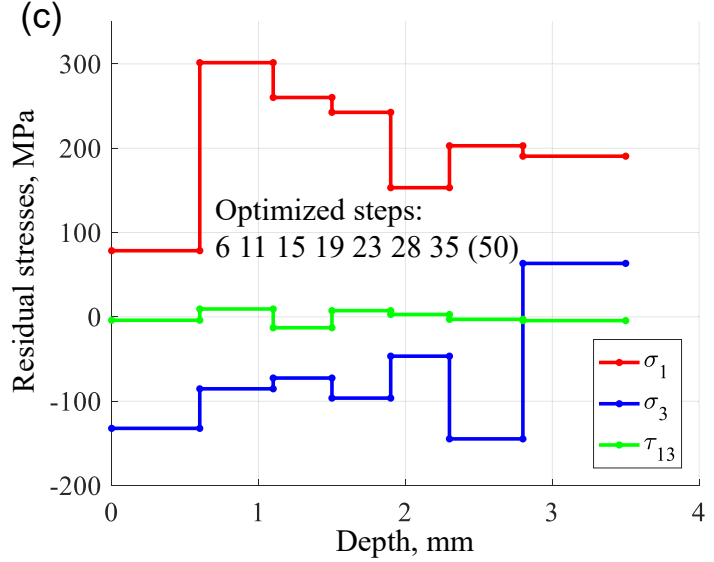

Figure 11. Ring-core experimental example, (a) relaxed strains with $0.1 \mathrm{~mm}$ incremental step, (b) residual stresses obtained with different depth steps: $0.5 \mathrm{~mm}$ and $1.0 \mathrm{~mm}$, (c) residual stresses obtained with the optimized steps.

elements. In order to make the procedure suitable for processing experimentally obtained relaxed strains, a method for deriving lower resolution coefficients was proposed by which coarser and also non-uniform step sequences can be analysed. A more general form of the integral method, and the related coefficient analysis, confirmed that first order expansions of the relaxed strains, as a function of groove eccentricity with respect to the rosette centre, are zero. As a consequence, the integral method with the combined strains and stresses can be considered accurate enough even if the ring-core is produced with an eccentricity entity typical of the correct experimental implementation of the technique, hence still by using the proposed high resolution coefficient matrices. An example of practical application of the obtained coefficients was finally presented. The residual stress distributions obtained with different depth steps, and in particular with an optimized non-uniform step sequence, were calculated and compared, thus demonstrating the applicability of the lower resolution coefficient determination procedure. 


\section{Acknowledgements}

Regione Toscana is acknowledged for funding this project: Bando 2009. Reg. (CE) n.1083/2006. POR CReO/FESR 2007-2013. Linea di intervento 1.3 a.

\section{References}

1. ASTM E837 - 13a. Standard Test Method for Determining Residual Stresses by the Hole-Drilling Strain-Gage Method, 2013.

2. P.V. Grant, J.D. Lord, and P.S. Whitehead. Standard Test Method for Determining Residual Stresses by the HoleDrilling Strain-Gage Method, 2002. National Physical Laboratory, Measurement Good Practice Guide No. 53, ISSN 1368-6550.

3. E. Procter and E.M. Beaney. Trepan or Ring Core Method, Centre-Hole Method, Sach's Method, Blind Hole Methods, Deep Hole Technique. Advances in Surface Treatments, 4:165-198, 1987.

4. W. Böhm, E. Stücker, and H. Wolf. Principles and potential applications of the ring-core method for determining residual stresses. $R A M, 4: 5-10,1988$.

5. A. Ajovalasit, G. Petrucci, and B. Zuccarello. Determination of nonuniform residual stresses using the ring-core method. Journal of Engineering Materials and Technology, Transactions of the ASME, 118(2):224-228, 1996.

6. B. Zuccarello. Optimization of depth increment distribution in the ring-core method. Journal of Strain Analysis for Engineering Design, 31(4):251-258, 1996.

7. F. Menda, F. Trebuňa, and P. Šarga. Determination of the necessary geometric parameters of the specimen in RingCore method. Applied Mechanics and Materials, 486:90-95, 2014.

8. F. Menda, P. Šarga, and F. Trebuňa. Estimation of residual stress field uniformity when using the ring-core method. Advanced Materials Research, 996:325-330, 2014.

9. F. Menda, P. Šarga, T. Lipták, and F. Trebuňa. Analysis of the geometric shape of the cutter in Ring-Core measurement. Procedia Engineering, 96:289-293, 2014.

10. B. Zuccarello, F. Menda, and M. Scafidi. Error and uncertainty analysis of non-uniform residual stress evaluation by using the ring-core method. Experimental Mechanics, 56(9):1531-1546, 2016.

11. A.J.G. Lunt, E. Salvati, L. Ma, I.P. Dolbyna, T.K. Neo, and A.M. Korsunsky. Full in-plane strain tensor analysis using the microscale ring-core FIB milling and DIC approach. Journal of the Mechanics and Physics of Solids, 94:47-67, 2016.

12. E. Salvati, T. Sui, A.J.G. Lunt, and A.M. Korsunsky. The effect of eigenstrain induced by ion beam damage on the apparent strain relief in FIB-DIC residual stress evaluation. Materials and Design, 92:649-658, 2016.

13. M. Beghini, L. Bertini, and L.F. Mori. Evaluating non-uniform residual stress by the hole-drilling method with concentric and eccentric holes. Part I. Definition and validation of the influence functions. Strain, 46:324-336, 2010.

14. M. Beghini, L. Bertini, and L.F. Mori. Evaluating non-uniform residual stress by the hole-drilling method with concentric and eccentric holes. Part II: Application of the influence functions to the inverse problem. Strain, 46:337346,2010

15. M. Beghini, L. Bertini, and C. Santus. A procedure for evaluating high residual stresses using the blind hole drilling method, including the effect of plasticity. Journal of Strain Analysis for Engineering Design, 45:301-318, 2010. 
16. M. Beghini, C. Santus, E. Valentini, and A. Benincasa. Experimental verification of the hole drilling plasticity effect correction. Materials Science Forum, 681:151-158, 2011.

17. A. Nau and B. Scholtes. Evaluation of the High-Speed Drilling Technique for the Incremental Hole-Drilling Method. Experimental Mechanics, 53:531-542, 2013.

18. A. Nau, D. von Mirbach, and B. Scholtes. Improved Calibration Coefficients for the Hole-Drilling Method Considering the Influence of the Poisson Ratio. Experimental Mechanics, 53:1371-1381, 2013.

19. P. Šarga and F. Menda. Comparison of Ring-Core Method and Hole-drilling Method Used for Determining Residual Stresses. American Journal of Mechanical Engineering, 1(7):335-338, 2013.

20. J. Václavík, O. Weinberg, P. Bohdan, J. Jankovec, and S. Holý. Evaluation of Residual Stresses using Ring Core Method. In EPJ Web of Conferences, ICEM 14 - 14th International Conference on Experimental Mechanics, volume 6 of 44004, pages 1-6, 2010.

21. A. Giri and M.M. Mahapatra. On the measurement of sub-surface residual stresses in SS 304L welds by dry ring core technique. Measurement, 106:152-160, 2017.

22. L. Xu, H. Zhao, H. Jing, and Y. Han. Finite element analysis of calibration coefficients for residual stress measurements by the ring core procedure. Materialpruefung/Materials Testing, 56(11-12):923-928, 2014.

23. A. Civín and M. Vlk. Ring-Core Residual Stress Measurement: Analysis of Calibration Coefficients for Incremental Strain Method. Bulletin of Applied Mechanics, 6:77-83, 2010.

24. A. Civín and M. Vlk. Determination of principal residual stresses' directions by incremental strain method. Applied and Computational Mechanics, 5:5-14, 2011.

25. M. Barsanti, M. Beghini, L. Bertini, B.D. Monelli, and C. Santus. First-order correction to counter the effect of eccentricity on the hole-drilling integral method with strain-gage rosettes. Journal of Strain Analysis for Engineering Design, 51(6):431-443, 2016.

26. G.S. Schajer. Measurement of non-uniform residual stresses using the hole-drilling method. Part I. Stress calculation procedures. Journal of Engineering Materials and Technology, Transactions of the ASME, 110:338-343, 1988.

27. G.S. Schajer. Measurement of non-uniform residual stresses using the hole-drilling method. Part II. Practical application of the integral method. Journal of Engineering Materials and Technology, Transactions of the ASME, 110:344-349, 1988.

28. M. Beghini, L. Bertini, C. Santus, A. Benincasa, L. Bertelli, and E. Valentini. Validazione sperimentale di una rosetta a 6 griglie per ridurre l'errore di eccentricità nella misura delle tensioni residue. In XXXIX congresso AIAS, 2010. AIAS 2010 - 067.

29. P. Iurea, C. Carausu, C. Tampu, B. Chirita, and V. Husanu. Residual stresses generated at roughing grinding and hard turning of raceways of bearing rings. International Journal of Modern Manufacturing Technologies, 8(2):19-24, 2016.

30. E. Valentini, A. Benincasa, and L. Bertelli. An automatic system for measuring residual stresses by the ring-core method. In XL congresso AIAS, 2011. AIAS 2011 - 145.

31. M. Barsanti, M. Beghini, C. Santus, A. Benincasa, and L. Bertelli. Integral method coefficients and regularization procedure for the ring-core residual stress measurement technique. In 9th European Conference on Residual Stresses, 2014. ECRS9, paper 242. 


\section{Appendix I}

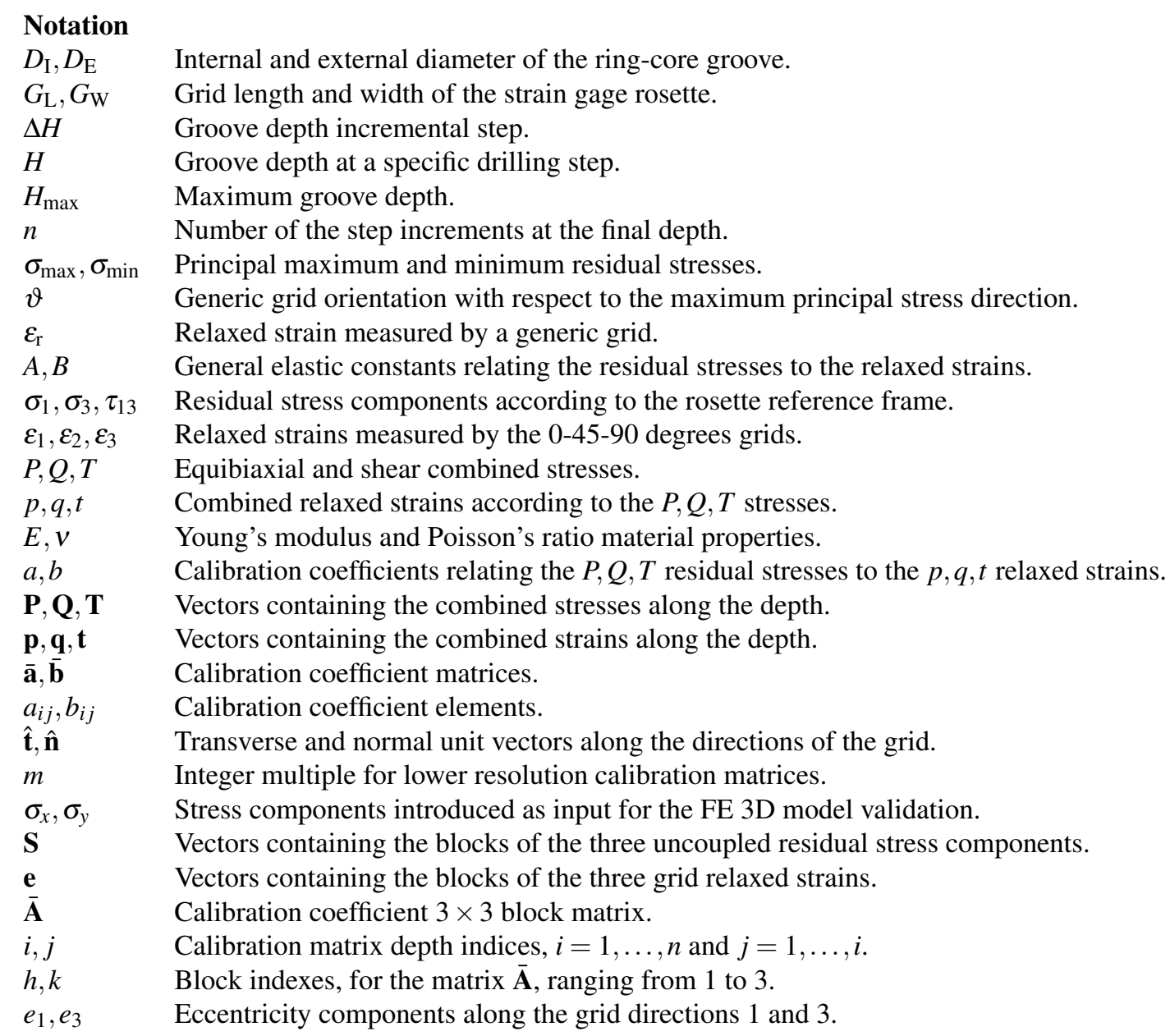


Online Appendix:

M. Barsanti, M. Beghini, C. Santus, A. Benincasa, L. Bertelli

Integral method coefficients for the ring-core technique to evaluate non-uniform residual stresses

The Journal of Strain Analysis for Engineering Design

Coefficients for $D_{\mathrm{I}}=14 \mathrm{~mm}, D_{\mathrm{E}}=18 \mathrm{~mm}, G_{\mathrm{L}}=5.0 \mathrm{~mm}, G_{\mathrm{W}}=2.5 \mathrm{~mm}$, maximum depth $H_{\max }=5.0 \mathrm{~mm}$ and depth resolution $\Delta H=0.1 \mathrm{~mm}$ $a_{i j} \times 10^{3}$

$$
b_{i j} \times 10^{3}
$$

$a_{1} \underset{5.3 .156}{5.3200} 4.5000$

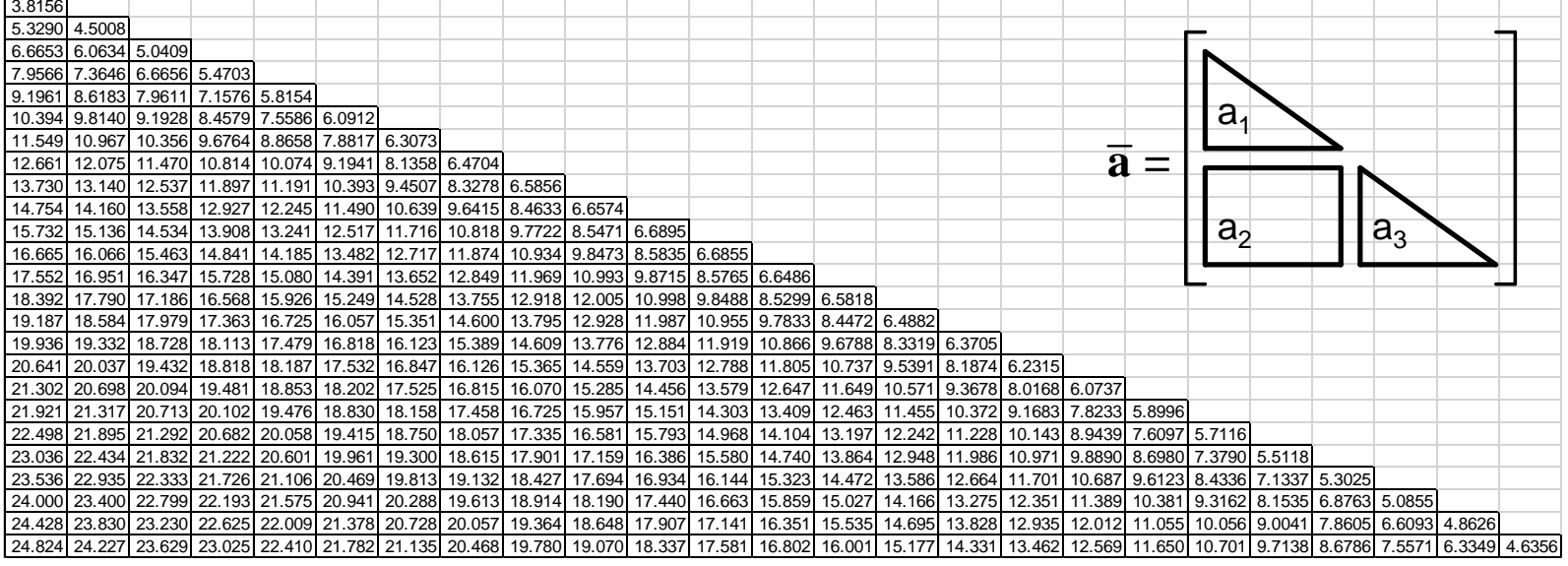

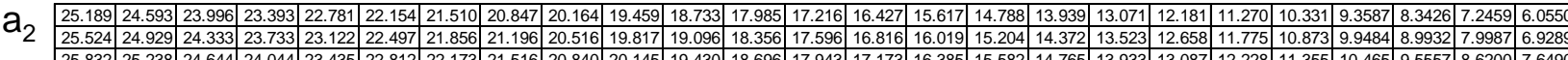

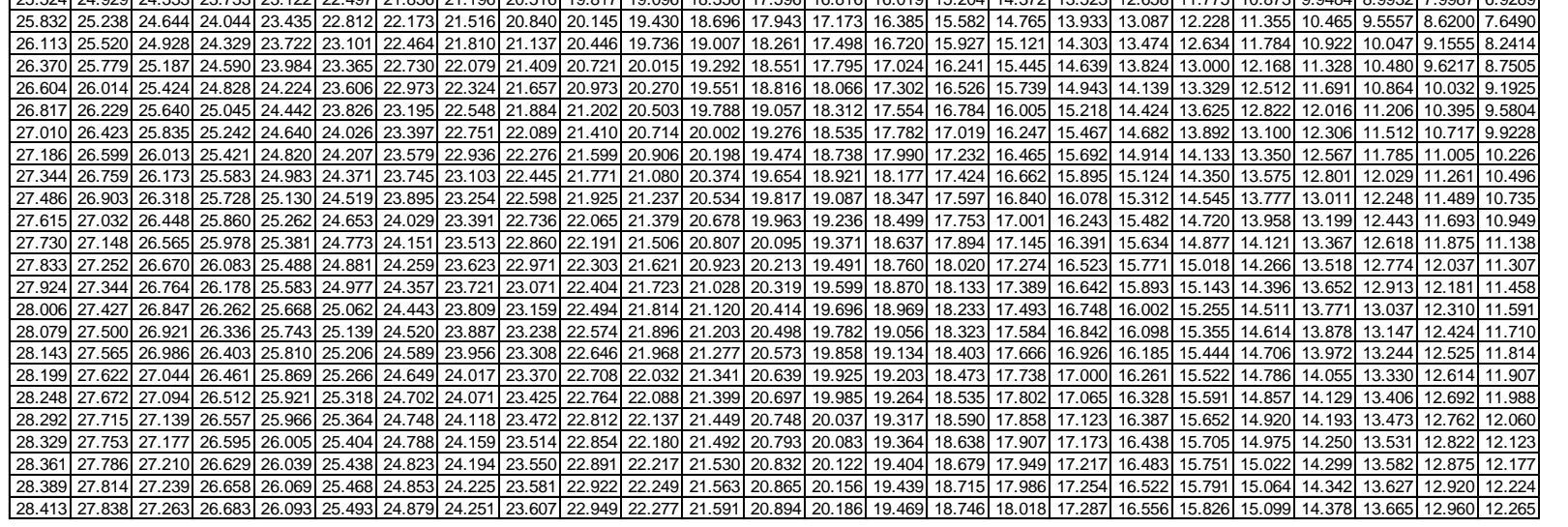

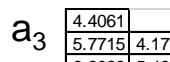

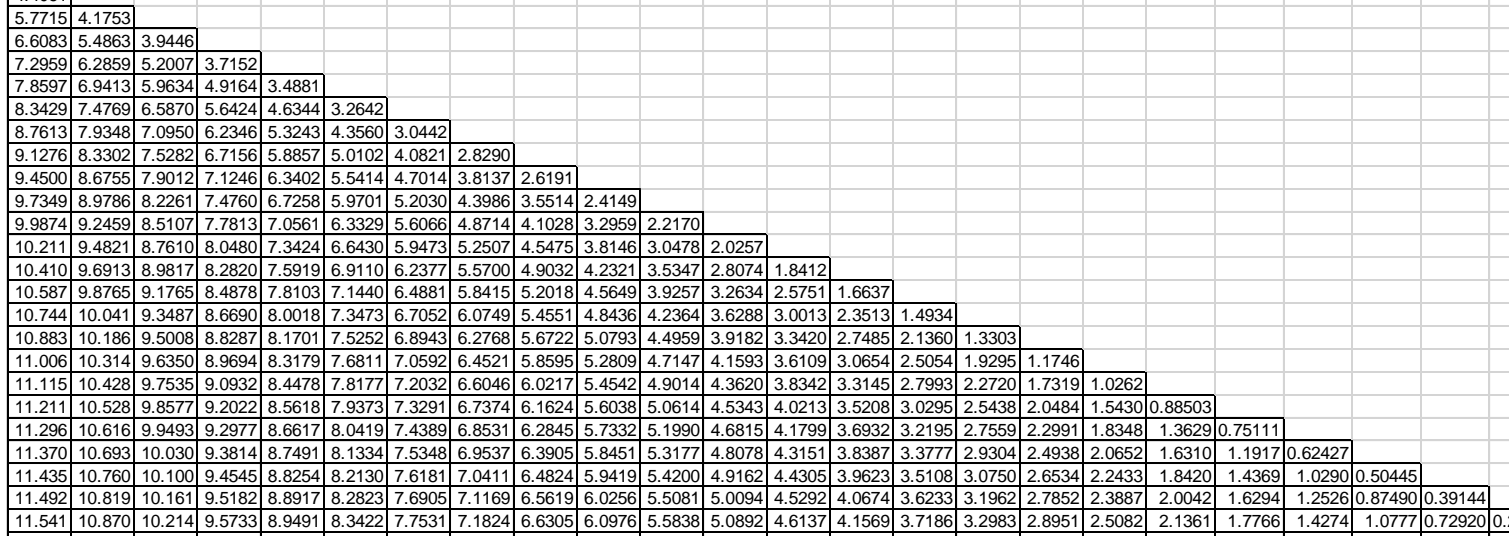

$b_{1} \frac{5}{6}$

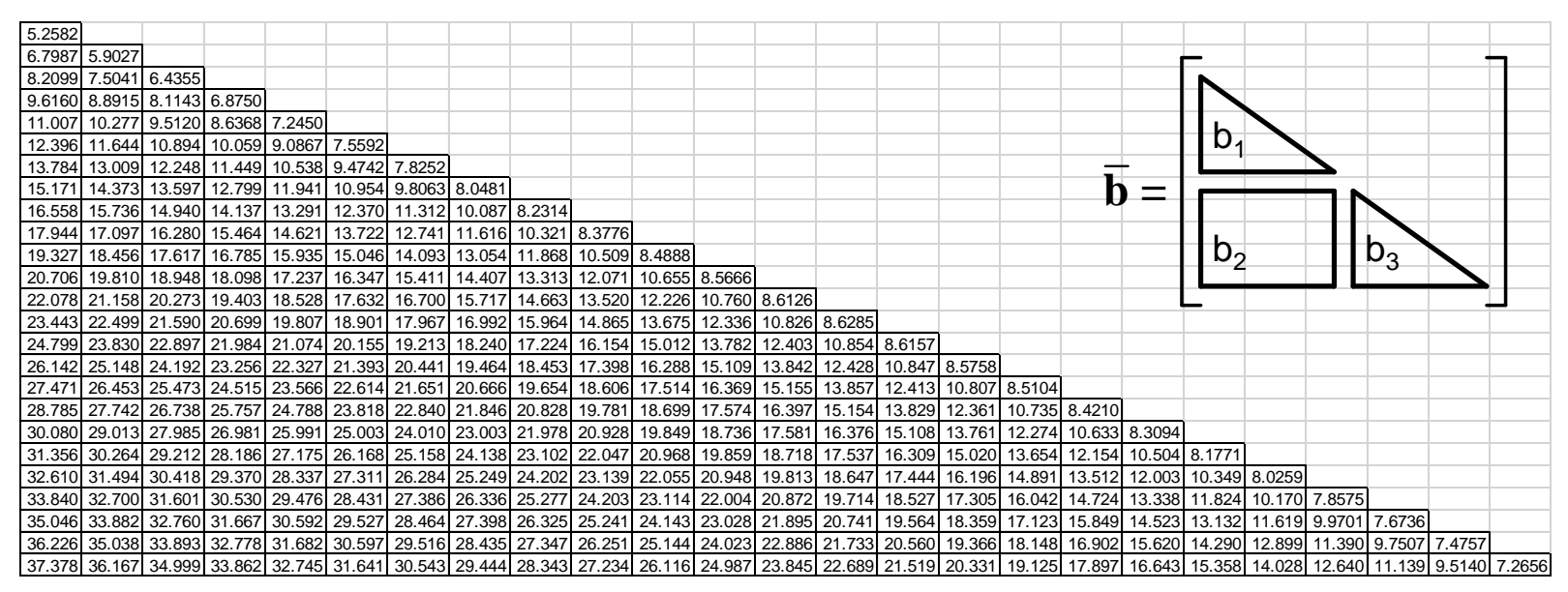

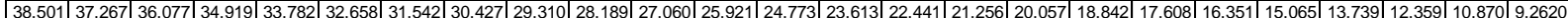

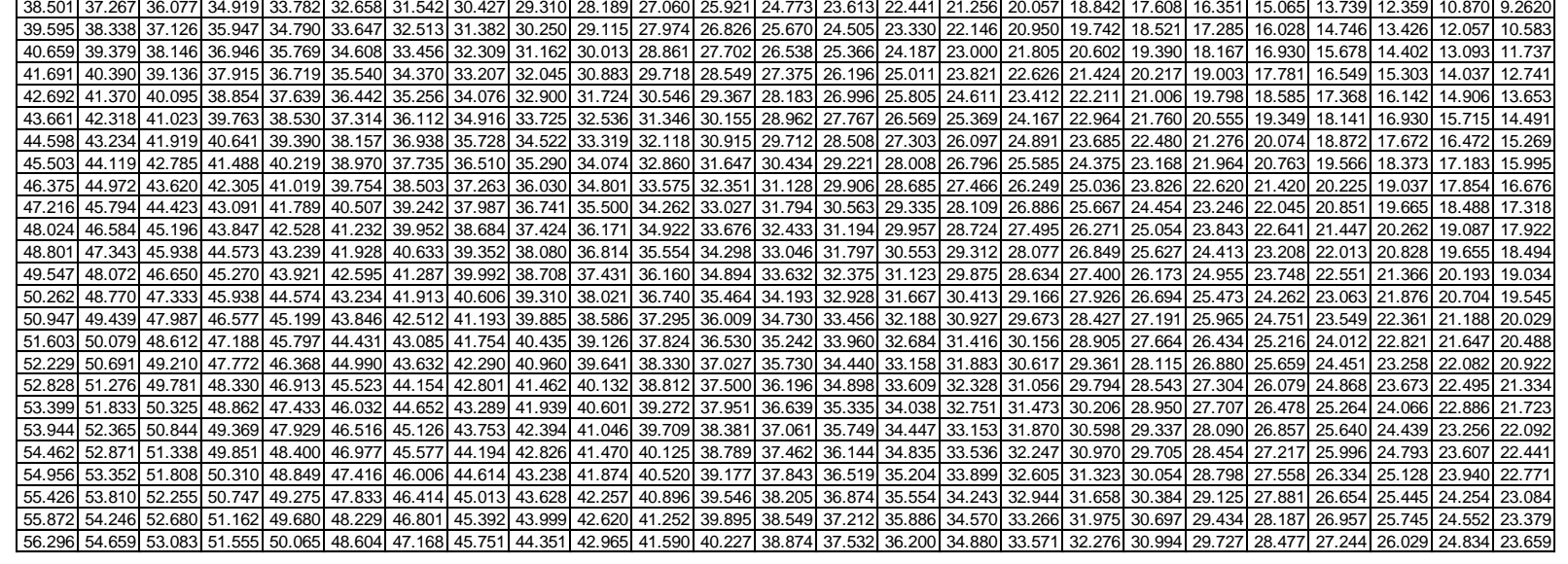

$b_{3}{ }_{8.09496}$

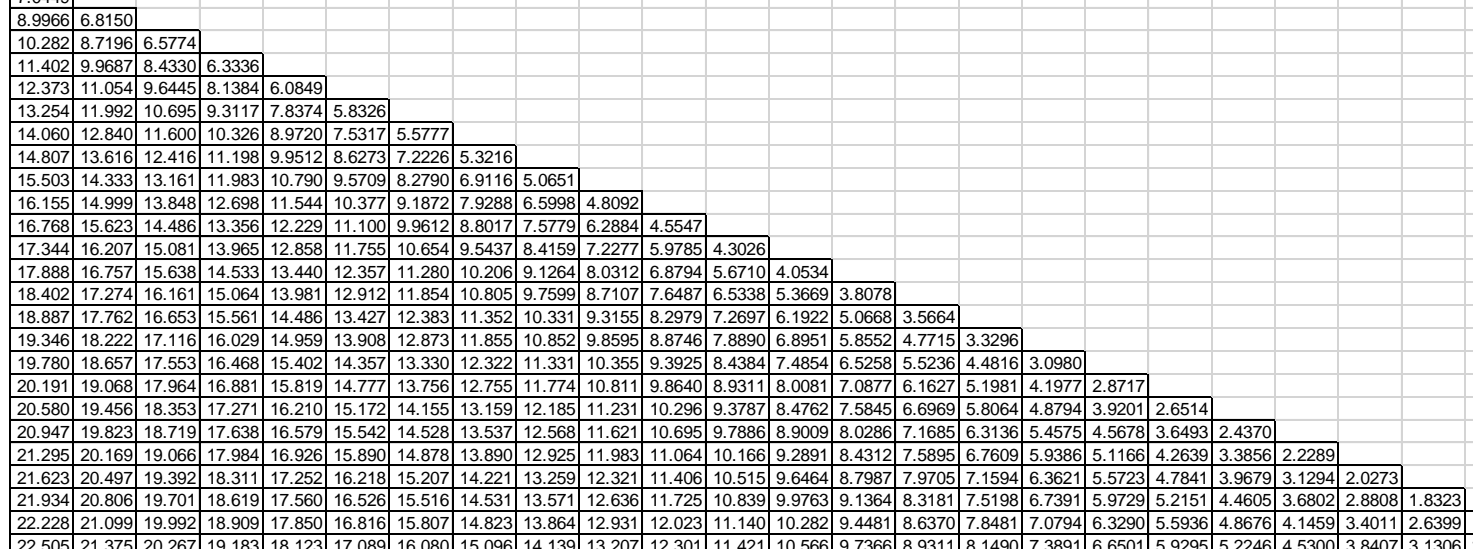

